\title{
A small note on the scaling of symmetric positive definite semiseparable matrices
}

\author{
Raf Vandebril, Gene Golub, and Marc Van Barel
}

\author{
Raf Vandebril \\ Department of Computer Science \\ KU Leuven, Belgium \\ Raf.Vandebrilecs.kuleuven.be
}

Gene Golub

SCCM Program, Stanford University Stanford, California, USA

Marc Van Barel

Department of Computer Science

KU Leuven, Belgium

Marc.VanBarel@cs.kuleuven.be

\begin{abstract}
In this paper we will adapt a known method for diagonal scaling of symmetric positive definite tridiagonal matrices towards the semiseparable case. Based on the fact that a symmetric, positive definite tridiagonal matrix $\mathrm{T}$ satisfies property A, one can easily construct a diagonal matrix (D) over cap such that (D) over capT (D) over cap has the lowest condition number over all matrices DTD, for any choice of diagonal matrix D. Knowing that semiseparable matrices are the inverses of tridiagonal matrices, one can derive similar properties for semiseparable matrices. Here, we will construct the optimal diagonal scaling of a semiseparable matrix, based on a new inversion formula for semiseparable matrices. Some numerical experiments are performed. In a first experiment we compare the condition numbers of the semiseparable matrices before and after the scaling. In a second numerical experiment we compare the scalability of matrices coming from the reduction to semiseparable form and matrices coming from the reduction to tridiagonal form.
\end{abstract}

\section{Article information}

- Vandebril, Raf; Golub, G.; Van Barel, Marc. A small note on the scaling of symmetric positive definite semiseparable matrices, Numerical Algorithms, volume 41, issue 3, pages 319-326, 2006.

- The content of this article is identical to the content of the published paper, but without the final typesetting by the publisher.

- Journal's homepage: http://link.springer.com/journal/11075

- Published version: http://dx.doi.org/10.1007/s11075-006-9014-x

- KU Leuven's repository url: https://lirias.kuleuven.be/handle/123456789/123791

\section{KU LEUVEN}




\title{
A small note on the scaling of symmetric positive definite semiseparable matrices ${ }^{* \dagger}$
}

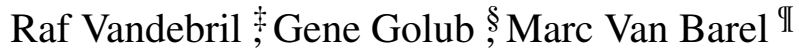 \\ February 18, 2014
}

\begin{abstract}
In this paper we will adapt a known method for diagonal scaling of symmetric positive definite tridiagonal matrices towards the semiseparable case. Based on the fact that a symmetric, positive definite tridiagonal matrix $T$ satisfies property A, one can easily construct a diagonal matrix $\hat{D}$ such that $\hat{D} T \hat{D}$ has the lowest condition number over all matrices $D T D$, for any choice of diagonal matrix $D$. Knowing that semiseparable matrices are the inverses of tridiagonal matrices, one can derive similar properties for semiseparable matrices. Here, we will construct the optimal diagonal scaling of a semiseparable matrix, based on a new inversion formula for semiseparable matrices.

Some numerical experiments are performed. In a first experiment we compare the condition numbers of the semiseparable matrices before and after the scaling. In a second numerical experiment we compare the scalability of matrices coming from the reduction to semiseparable form and matrices coming from the reduction to tridiagonal form.

Keywords: Condition number, semiseparable matrices, property A, inversion of semiseparable matrices

AMS Classification: 65F35, 15A12
\end{abstract}

\section{Introduction}

The matrices we consider in this paper are symmetric and positive definite. A symmetric matrix $A$ is said to have property $A$ when there exists a permutation $P$, such that $P A P^{T}$ is of the following form:

$$
\hat{A}=P A P^{T}=\left(\begin{array}{cc}
D_{1} & F \\
F^{T} & D_{2}
\end{array}\right),
$$

where $F$ is an arbitrary matrix and $D_{1}$ and $D_{2}$ are diagonal matrices. As the matrix is symmetric and positive definite the elements of $D_{1}$ and $D_{2}$ are positive. These matrices are known to be scaled best if the diagonal matrices $D_{1}$ and $D_{2}$ are equal to the identity matrix (see $[2,1,5]$ ). This can be achieved rather easily by applying a diagonal scaling of the matrix. Defining the scaling matrix $\hat{D}$ as

$$
\hat{D}=\left(\begin{array}{cc}
\frac{1}{\sqrt{\left(D_{1}\right)_{i, i}}} & \\
& \frac{1}{\sqrt{\left(D_{2}\right)_{i, i}}}
\end{array}\right)
$$

\footnotetext{
*The authors wish to thank Nicola Mastronardi, for bringing the subject of semiseparable matrices to their attention.

${ }^{\dagger}$ The research was partially supported by the Research Council K.U.Leuven, project OT/00/16 (SLAP: Structured Linear Algebra Package), by the Fund for Scientific Research-Flanders (Belgium), projects G.0078.01 (SMA: Structured Matrices and their Applications), G.0176.02 (ANCILA: Asymptotic aNalysis of the Convergence behavior of Iterative methods in numerical Linear Algebra), G.0184.02 (CORFU: Constructive study of Orthogonal Functions) and G.0455.0 (RHPH: Riemann-Hilbert problems, random matrices and Padé-Hermite approximation), and by the Belgian Programme on Interuniversity Poles of Attraction, initiated by the Belgian State, Prime Minister's Office for Science, Technology and Culture, project IUAP V-22 (Dynamical Systems and Control: Computation, Identification \& Modelling). The scientific responsibility rests with the authors. The second author participates in the SCCM program, Gates 2B, Stanford University, CA, USA and is also partially supported by the NSF. The first author visited the second one with a grant by the Fund for Scientific Research-Flanders (Belgium).

${ }^{\ddagger}$ raf.vandebril@cs.kuleuven.ac.be, Dept. Computerwetenschappen, Celestijnenlaan 200A, 3001 Heverlee (Leuven), Belgium.

§golub@stanford.edu, Dept. of Computer Science, Gates Building 2B, Stanford, CA 94305-9025, USA.

Imarc.vanbarel@cs.kuleuven.ac.be, Dept. Computerwetenschappen, Celestijnenlaan 200A, 3001 Heverlee (Leuven), Belgium.
} 
gives us the desired solution. In this paper we will use this result for tridiagonal matrices to derive the best diagonal scaling for semiseparable matrices. A tridiagonal matrix satisfies property A. For the permutation $P^{T}$ one uses a reshuffling of the columns, thereby first placing the even columns $([2,4, \ldots])$ and after the even, the odd columns $([1,3,5, \ldots])$. Applying the same reshuffling technique $P$ for the rows, one gets a matrix $P T P^{T}$ of the desired form.

The optimal scaling matrix $\hat{D}$ for semiseparable matrices is calculated by using an order $O(n)$ inversion formula for semiseparable matrices. The most early paper related to the inversion of semiseparable and tridiagonal matrices is [3], in which irreducible symmetric tridiagonal matrices are inverted. The most early papers related to the inversion of semiseparable matrices are [6,7]. These papers focus on semiseparable matrices arising in statistical applications. In fact inverting these matrices, as well as scaling these matrices is a very important subject in statistics. The scaling is related to the variance in weighted least squares regression coefficients [4] (in this paper attention is paid to the condition number of a specific semiseparable matrix, arising in statistical applications).

The present paper is organized as follows: in Section 2, we derive the structure of the optimal diagonal scaling matrix for semiseparable matrices. In Section 3 we give a method for computing the inverse of a semiseparable matrix. This method can now be used for calculating the optimal scaling matrix. In Section 4 we present numerical experiments showing the impact of the scaling on the condition number and on the accuracy when solving linear systems with semiseparable matrices as coefficient matrices.

\section{The scaling technique}

Using the knowledge related to matrices having property A as described in the introduction, the optimal diagonal scaling of a tridiagonal matrix $T$ can be obtained by choosing the diagonal $\hat{D}$ in such a way that the diagonal elements of the tridiagonal matrix $\hat{D} T \hat{D}$ are equal to one. This means that for a tridiagonal matrix $T$ with diagonal elements $a_{i}$, our optimal scaling diagonal matrix $\hat{D}$ has as diagonal elements $1 / \sqrt{a_{i}}$. This is well defined as a symmetric positive definite matrix has positive diagonal elements.

Defining the condition number $\kappa$ of a matrix $A$ as $\kappa(A)=\|A\|\left\|A^{-1}\right\|$ for any consistent matrix norm, we have that $\kappa(A)=\kappa\left(A^{-1}\right)$. This means that for an invertible symmetric, positive definite tridiagonal matrix $T$, with inverse $S$ and optimal diagonal scaling matrix $\hat{D}$ for $T$, we have:

$$
\kappa\left(\hat{D}^{-1} S \hat{D}^{-1}\right)=\kappa(\hat{D} T \hat{D}) \leq \kappa(D T D)=\kappa\left(D^{-1} S D^{-1}\right),
$$

for any matrix $D$. Using the inversion formula for semiseparable matrices as presented in Section 3 we can easily calculate in $O(n)$ flops the optimal diagonal scaling of a semiseparable matrix.

\section{Inversion of semiseparable matrices}

Traditional inversion methods of semiseparable matrices are based on the generator representation. This means that the lower triangular part of a semiseparable matrix, as well as the upper triangular part are coming from rank 1 matrices. Based on the Givens-vector representation (see [9]), we will derive here an inversion formula for the class of semiseparable matrices representable in this way. Moreover, this class is more general than the class of generator representable matrices (see [9]). The algorithm will be designed for a $4 \times 4$ semiseparable matrix, as this illustrates the general case. Our semiseparable matrix $S$ is of the following form (see [9]):

$$
S=\left(\begin{array}{cccc}
c_{1} d_{1} & c_{2} s_{1} d_{1} & c_{3} s_{2} s_{1} d_{1} & s_{3} s_{2} s_{1} d_{1} \\
c_{2} s_{1} d_{1} & c_{2} d_{2} & c_{3} s_{2} d_{2} & s_{3} s_{2} d_{2} \\
c_{3} s_{2} s_{1} d_{1} & c_{3} s_{2} d_{2} & c_{3} d_{3} & s_{3} d_{3} \\
s_{3} s_{2} s_{1} d_{1} & s_{3} s_{2} d_{2} & s_{3} d_{3} & d_{4}
\end{array}\right)
$$

This matrix is stored by using $3 n-2$ parameters namely the $c_{i}, s_{i}$ and the $d_{i}\left(d_{1} \neq 0\right.$ as the matrix is invertible). Note however that the $c_{i}$ and $s_{i}$ represent the cosine and sine of the same angle, this means that 
essentially $2 n-1$ parameters are needed to represent this matrix. (More information can be found in [9]). The inverse of the semiseparable matrix $S$ is denoted as $T$ :

$$
T=\left(\begin{array}{cccc}
a_{1} & b_{1} & & \\
b_{1} & a_{2} & b_{2} & \\
& b_{2} & a_{3} & b_{3} \\
& & b_{3} & a_{4}
\end{array}\right)
$$

Let us start by calculating $a_{1}$ and $b_{1}$. The 4 equations corresponding to the first column of the product $S T=I$, namely $S T e_{1}=e_{1}$ (the elements $e_{i}$ represent the standard basis vectors), are:

$$
a_{1}\left(c_{1} d_{1}\right)+b_{1}\left(c_{2} s_{1} d_{1}\right)=1
$$

whereas, the remaining equations collapse into one singular equation: (This happens because the two first columns of $S$ are dependent below the first row.)

$$
a_{1}\left(s_{1} d_{1}\right)+b_{1} d_{2}=0 .
$$

Rewriting equation (3) towards $a_{1} d_{1}$ we get

$$
a_{1} d_{1}=\frac{-b_{1} d_{2}}{s_{1}} .
$$

If $s_{1}=0$ we can easily see that $b_{1}=0$ and $a_{1}=1 / d_{1}$, so let us assume $s_{1}$ to be different from zero.(We note that by definition of the representation the cosines are always positive. If $s_{1}=0$ this implies $c_{1}=1$.) Substituting $a_{1} d_{1}$ in equation (2) and rewriting the equation towards $b_{1}$ gives us

$$
b_{1}=\frac{1}{c_{2} s_{1} d_{1}-d_{2} \frac{c_{1}}{s_{1}}} .
$$

Note that this equation is well defined as $s_{1}$ is different from zero and an easy calculation reveals that also the denominator in the equation has to be different from zero, otherwise the semiseparable matrix would be singular. Once we have $b_{1}$ the calculation of $a_{1}$ is straightforward using equation (3). We have calculated now $a_{1}$ and $b_{1}$. To continue we calculate $a_{2}$ and $b_{2}$. We write down the equations corresponding to the second column of $S T=I$, i.e. $S T e_{2}=e_{2}$, let us consider the equations on and below the diagonal. This gives us:

$$
e_{2}^{T} S T e_{2}=b_{1}\left(c_{2} s_{1} d_{1}\right)+a_{2}\left(c_{2} d_{2}\right)+b_{2}\left(c_{3} s_{2} d_{2}\right)=1,
$$

while the equations $e_{3}^{T} S T e_{2}=0$ and $e_{4}^{T} S T e_{2}=0$ collapse again into one single equation:

$$
b_{1}\left(s_{2} s_{1} d_{1}\right)+a_{2}\left(s_{2} d_{2}\right)+b_{2} d_{3}=0 .
$$

We will distinguish between two cases now: $s_{2}=0$ and $s_{2} \neq 0$.

- Let us assume $s_{2}=0$. This implies directly that $b_{2}=0$. Again we have to distinguish between two cases to calculate $a_{2}$. Assuming $s_{1}=0$ we get $a_{2}=1 / d_{2}$.

Assuming now $s_{1} \neq 0$, we can write down equation $e_{1}^{T} S T e_{2}=0$ :

$$
b_{1}\left(c_{1} d_{1}\right)+a_{2}\left(c_{2} s_{1} d_{1}\right)=0 .
$$

Rewriting this equation towards $a_{2}$ we get $a_{2}=-b_{1} c_{1} /\left(c_{2} s_{1}\right)$. This means that for $s_{2}=0$ we can calculate $a_{2}$ and $b_{2}$.

- Let us assume now $s_{2} \neq 0$ and $f_{2}=b_{1} s_{1} d_{1}$, then we get for equations (4) and (5):

$$
\left\{\begin{aligned}
c_{2} f_{2}+a_{2}\left(c_{2} d_{2}\right)+b_{2}\left(c_{3} s_{2} d_{2}\right) & =1 \\
s_{2} f_{2}+a_{2}\left(s_{2} d_{2}\right)+b_{2} d_{3} & =0
\end{aligned}\right.
$$


Extracting $a_{2} d_{2}$ from the second equation of (6) gives us:

$$
a_{2} d_{2}=\frac{-b_{2} d_{3}-s_{2} f_{2}}{s_{2}}
$$

(which is well defined as $s_{2} \neq 0$ ) filling it in in the first equation of (6), we get:

$$
b_{2}=\frac{1}{c_{3} s_{2} d_{2}-d_{3} \frac{c_{2}}{s_{2}}}
$$

which is similar to equation (3). The calculation of $a_{2}$ is again a little more complicated. Two cases can occur:

- If $d_{2}=0$ we use the equation $e_{1} S T e_{2}=0$ and extract $a_{2}$ from this equation, this gives us:

$$
a_{2}=\frac{-b_{2} c_{3} s_{2} s_{1}-b_{1} c_{1}}{c_{2} s_{1}} .
$$

We know that $c_{2} s_{1}$ has to be different from zero, otherwise the semiseparable matrix would have been singular.

- If $d_{2} \neq 0$ we use equation (5). Giving us

$$
a_{2}=\frac{-b_{2} d_{3}-s_{2} f_{2}}{s_{2} d_{2}}
$$

which is well defined because both $s_{2}$ and $d_{2}$ are different from zero.

This last procedure can similarly be repeated for the third column, leading to the same formula's for calculating $a_{3}$ and $b_{3}$. Only the last column needs some extra attention. For the last column we consider the equations $e_{4}^{T} S T e_{4}=1$ and $e_{3}^{T} S T e_{4}=0$ :

$$
\left\{\begin{aligned}
b_{3}\left(s_{3} d_{3}\right)+a_{4} d_{4} & =1 \\
b_{3}\left(c_{3} d_{3}\right)+a_{4}\left(s_{3} d_{3}\right) & =0
\end{aligned}\right.
$$

Using these formulas we get

$$
a_{4}=\frac{1-b_{3} s_{3} d_{3}}{d_{4}},
$$

if $d_{4} \neq 0$ or else if $d_{4}=0$

$$
a_{4}=-\frac{b_{3} c_{3}}{s_{3}}
$$

because if $d_{4}=0, s_{3}$ has to be different from zero, otherwise the matrix would have been singular.

Even though we calculated only the inverse of a $4 \times 4$ matrix, the procedure clearly demonstrates how to calculate inverses of larger semiseparable matrices. Moreover, inverting semiseparable matrices in this way leads to an $O(n)$ procedure.

\section{Numerical experiments}

The software used for performing these numerical experiments can be downloaded from: http://www.cs.kuleuven.ac.be/ marc.

The experiments were performed using Matlab 7 Release 14. ${ }^{1}$.

For the first set of experiments we generated matrices of sizes ranging from 200 to 600 , thereby varying the condition number from $10^{8}$ to $10^{14}$. The table below shows the difference in condition number, with and without the diagonal scaling.

\footnotetext{
${ }^{1}$ Matlab is a registered trademark of The Mathworks, inc.
} 


\begin{tabular}{|c|c|c|c|c|c|c|}
\hline$\kappa \approx$ & size $\longrightarrow$ & 200 & 300 & 400 & 500 & 600 \\
\hline \multirow[t]{2}{*}{$10^{8}$} & without scaling & $1.775 \mathrm{e}+08$ & $9.197 e+07$ & $7.837 e+07$ & $7.803 e+07$ & $7.720 \mathrm{e}+07$ \\
\hline & with scaling & $2.783 e+06$ & $1.515 \mathrm{e}+07$ & $7.528 \mathrm{e}+06$ & $1.449 \mathrm{e}+07$ & $7.874 \mathrm{e}+06$ \\
\hline \multirow[t]{2}{*}{$10^{10}$} & without scaling & $1.319 \mathrm{e}+10$ & $1.151 \mathrm{e}+10$ & $1.012 \mathrm{e}+10$ & $7.463 e+09$ & $6.387 e+09$ \\
\hline & with scaling & $3.069 \mathrm{e}+08$ & $1.325 \mathrm{e}+09$ & $4.186 \mathrm{e}+08$ & $5.071 \mathrm{e}+08$ & $2.344 \mathrm{e}+08$ \\
\hline \multirow[t]{2}{*}{$10^{12}$} & without scaling & $1.344 \mathrm{e}+12$ & $1.432 \mathrm{e}+12$ & $9.865 e+11$ & $1.065 \mathrm{e}+12$ & $8.168 \mathrm{e}+11$ \\
\hline & with scaling & $1.846 \mathrm{e}+10$ & $7.043 \mathrm{e}+09$ & $5.135 e+10$ & $2.337 e+10$ & $4.045 \mathrm{e}+10$ \\
\hline \multirow[t]{2}{*}{$10^{14}$} & without scaling & $1.259 \mathrm{e}+14$ & $1.673 e+14$ & $6.909 e+13$ & $1.079 \mathrm{e}+14$ & $6.556 e+13$ \\
\hline & with scaling & $2.466 e+11$ & $2.209 e+12$ & $1.942 \mathrm{e}+12$ & $9.188 \mathrm{e}+12$ & $6.571 \mathrm{e}+11$ \\
\hline
\end{tabular}

Table 1: Scalability of semiseparable matrices.

Recently an orthogonal similarity reduction to semiseparable form was proposed [8]. This reduction performs $9 n^{2}$ more operations than the corresponding reduction to tridiagonal form. Both reductions have an overall cost of $O\left(n^{3}\right)$. These $9 n^{2}$ operations create an extra convergence behavior (nested subspace iteration) inside the reduction algorithm. This behavior is not present in the reduction to tridiagonal form. In a second experiment we compared the scalability of the matrices reduced to either tridiagonal or semiseparable form. Positive definite matrices of various condition numbers were generated and afterwards reduced to semiseparable and tridiagonal form. For both forms the scalability was tested. The results show the condition numbers of the matrices after the reduction without scaling, and then after scaling was applied. In the table with $S$ without we denote the semiseparable matrix without scaling, with $T$ without, the tridiagonal without scaling, and with $S$ with or $T$ with, these matrices with scaling.

\begin{tabular}{||l|l||r|r|r|r|r||}
\hline \hline$\kappa \approx$ & size $\longrightarrow$ & 200 & 300 & 400 & 500 & 600 \\
\hline $10^{8}$ & $S$ without & $1.608 \mathrm{e}+08$ & $6.012 \mathrm{e}+07$ & $9.702 \mathrm{e}+07$ & $7.306 \mathrm{e}+07$ & $5.759 \mathrm{e}+07$ \\
& $S$ with & $2.044 \mathrm{e}+00$ & $2.156 \mathrm{e}+00$ & $2.046 \mathrm{e}+00$ & $2.214 \mathrm{e}+00$ & $2.017 \mathrm{e}+00$ \\
& $T$ without & $1.608 \mathrm{e}+08$ & $6.012 \mathrm{e}+07$ & $9.702 \mathrm{e}+07$ & $7.306 \mathrm{e}+07$ & $5.759 \mathrm{e}+07$ \\
& $T$ with & $1.070 \mathrm{e}+06$ & $3.940 \mathrm{e}+06$ & $1.096 \mathrm{e}+07$ & $4.363 \mathrm{e}+06$ & $6.862 \mathrm{e}+06$ \\
\hline $10^{10}$ & $S$ without & $1.482 \mathrm{e}+10$ & $7.719 \mathrm{e}+09$ & $7.892 \mathrm{e}+09$ & $1.261 \mathrm{e}+10$ & $7.448 \mathrm{e}+09$ \\
& $S$ with & $1.893 \mathrm{e}+00$ & $2.190 \mathrm{e}+00$ & $1.954 \mathrm{e}+00$ & $1.970 \mathrm{e}+00$ & $1.890 \mathrm{e}+00$ \\
& $T$ without & $1.482 \mathrm{e}+10$ & $7.719 \mathrm{e}+09$ & $7.892 \mathrm{e}+09$ & $1.261 \mathrm{e}+10$ & $7.448 \mathrm{e}+09$ \\
& $T$ with & $1.397 \mathrm{e}+08$ & $1.057 \mathrm{e}+09$ & $8.510 \mathrm{e}+08$ & $3.860 \mathrm{e}+08$ & $1.984 \mathrm{e}+08$ \\
\hline $10^{12}$ & $S$ without & $1.637 \mathrm{e}+12$ & $1.071 \mathrm{e}+12$ & $9.664 \mathrm{e}+11$ & $7.772 \mathrm{e}+11$ & $7.576 \mathrm{e}+11$ \\
& $S$ with & $2.160 \mathrm{e}+00$ & $1.905 \mathrm{e}+00$ & $1.809 \mathrm{e}+00$ & $2.115 \mathrm{e}+00$ & $1.898 \mathrm{e}+00$ \\
& $T$ without & $1.637 \mathrm{e}+12$ & $1.071 \mathrm{e}+12$ & $9.664 \mathrm{e}+11$ & $7.772 \mathrm{e}+11$ & $7.576 \mathrm{e}+11$ \\
& $T$ with & $7.110 \mathrm{e}+10$ & $2.697 \mathrm{e}+10$ & $5.145 \mathrm{e}+10$ & $1.937 \mathrm{e}+10$ & $1.213 \mathrm{e}+10$ \\
\hline $10^{14}$ & $S$ without & $1.344 \mathrm{e}+14$ & $1.394 \mathrm{e}+14$ & $1.617 \mathrm{e}+14$ & $8.475 \mathrm{e}+13$ & $8.471 \mathrm{e}+13$ \\
& $S$ with & $2.091 \mathrm{e}+00$ & $2.045 \mathrm{e}+00$ & $1.842 \mathrm{e}+00$ & $1.809 \mathrm{e}+00$ & $2.238 \mathrm{e}+00$ \\
& $T$ without & $1.637 \mathrm{e}+12$ & $1.071 \mathrm{e}+12$ & $9.664 \mathrm{e}+11$ & $7.772 \mathrm{e}+11$ & $7.576 \mathrm{e}+11$ \\
& $T$ with & $1.138 \mathrm{e}+12$ & $2.115 \mathrm{e}+12$ & $5.823 \mathrm{e}+11$ & $2.656 \mathrm{e}+12$ & $3.105 \mathrm{e}+12$ \\
\hline \hline
\end{tabular}

Table 2: Comparison in scalability.

This indicates that due to extra convergence properties of the reduction to semiseparable form [8], these matrices are far better scalable than the matrices coming from the reduction to tridiagonal form.

\section{Conclusions and future research}

In this paper an $O(n)$ scaling technique for semiseparable matrices was presented, based on the scaling of tridiagonal matrices. 
Future research will focus on a direct translation of property A towards the semiseparable case, as this could lead to scaling of more general classes of semiseparable matrices, such as sequentially or hierarchically semiseparable ones. Also attention will be paid towards scaling techniques for rectangular matrices, as this can improve accuracy e.g. in least squares problems.

\section{References}

[1] F. L. Bauer. Optimally scaled matrices. Numerische Mathematik, 5:73-87, 1963.

[2] G. E. Forsythe and E. G. Straus. On best conditioned matrices. Proceedings of the American Mathematical Society, 6:340-345, 1955.

[3] F. R. Gantmacher and M. G. Kreı̆n. Sur les matrices oscillatoires et complètement non négatives. Compositio Mathematica, 4:445-476, 1937.

[4] G. H. Golub. Comparison of the variance of minimum variance and weighted least squares regression coefficients. Annals of Mathematical Statistics, 34(3):984-991, September 1963.

[5] G. H. Golub and J. M. Varah. On the characterization of the best $L_{2}$-scaling of a matrix. SIAM Journal on Numerical Analysis, 11:472-479, 1974.

[6] S. N. Roy, B. G. Greenberg, and A. E. Sarhan. Evaluation of determinants, characteristic equations and their roots for a class of patterned matrices. Journal of the Royal Statistical Society. Series B. Methodological, 22:348-359, 1960.

[7] S. N. Roy and A. E. Sarhan. On inverting a class of patterned matrices. Biometrika, 43:227-231, 1956.

[8] M. Van Barel, R. Vandebril, and N. Mastronardi. An orthogonal similarity reduction of a matrix into semiseparable form. SIAM Journal on Matrix Analysis and its Applications, 27(1):176-197, 2005.

[9] R. Vandebril, M. Van Barel, and N. Mastronardi. A note on the representation and definition of semiseparable matrices. Numerical Linear Algebra with Applications, 12:839-858, June 2005. 